\title{
Prise en compte de la genèse d'un site alluvionnaire dans l'analyse de ses caractéristiques géotechniques : site de Cran (Ille et Vilaine)
}

\author{
Assia BAT ${ }^{(1),(2)}$, Jean-Claude BLIVET $T^{(1)}$, Daniel LEVACHER ${ }^{(2)}$ \\ (1) Laboratoire Régional des Ponts et Chaussées de Rouen, C.E.T.E Normandie - Centre. \\ Chemin de la poudrière B.P 24576121 Le Grand QUEVILLY Cedex \\ (2) UPRES A 6143 "Morphodynamique continentale et côtière", Groupe de Recherche \\ Génie Civil, Université de Caen, 24 rue des Tilleuls 14032 CAEN Cedex
}

\section{Résumé}

L'étude présentée dans cet article fait partie d'une série de travaux réalisés au laboratoire des ponts et chaussées de Rouen pour examiner l'influence des procédures de prélèvement et des procédures d'essais sur les caractéristiques de sol mesurées. Les résultats des essais œdométriques et des essais à l'appareil triaxial effectués sur l'argile molle française de Cran (site du pont de Cran : plaine de la Vilaine en aval de Redon), montrent une certaine dispersion des résultats due à la variabilité spatiale naturelle du sol en place. De façon implicite dans cette analyse, nous avons supposé que le matériau prélevé et ausculté est homogène. Si cette présupposition est relativement vraie à l'échelle globale du site, une analyse fine du sol montre qu'il le paraît moins dans le détail. Il s'agit généralement d'argile molle grise (dépôts marins récents). Aucune arrivée détritique plus grossière (sables) n'est observée dans les carottes. Par ailleurs, on peut distinguer trois types de discontinuités ou facteurs d'hétérogénéité : la présence de débris végétaux, la présence de discontinuités sédimentaires et la présence de plans de fracturations et même de glissements. Après une présentation générale du site les différentes discontinuités sont présentées pour tenter d'expliquer la dispersion des résultats qui peut être due à la variabilité spatiale du sol. L'analyse géologique faite sur le massif argileux de Cran illustre la difficulté d'étude des sols naturels. Elle remet en cause l'apparente homogénéité du site, et permet d'expliquer que des échantillons prélevés à la même profondeur peuvent avoir des propriétés physiques et mécaniques différentes. L'analyse géologique de la genèse du site permet donc une meilleure approche des caractéristiques géotechniques.

\section{Abstract}

The sampling operations and laboratory testing protocols influence the measurements of the soils characteristics. To determine these influences a large research program has been performed at the laboratory of "Ponts et chaussées" of Rouen. It was consisting in oedometer and triaxial tests on a very soft clay from the Brittany province at Cran in the Vilaine river plain near Redon city. A clear and significative variability of the geotechnical properties was found for this soft clay. It is natural spatial variability of the in situ soil. Considering the cored soft clay as an homogenous soil before all tests, this assumption is becoming not checked by the analysis of the laboratory tests results even if it is true for the soil at the large scale. In details and for a small scale it is totally different for this grey soft clay classifies as recent marine deposit. Any sandy fine layer wasn't found in the samples. So many heterogeneities has been observed. Three heterogeneity factors or discontinuity types has been identified as natural debris of plants, 
sedimentary discontinuities and fracture plans. After giving the informations about the site, the main geotechnical characteristics of the soft clay, all the types of the discontinuities are presented to explain the test results dispersion which can linked to the natural variability of the soil. A geological analysis made on the soft clay site of Cran shows clearly the difficulties to study the natural soils. The results of the tests confirm the non homogeneity of the site and the physical and mechanical properties differences for samples cored at the same depth. An geological analysis of the genes allows a better approach of the geotechnical characteristics.

\section{Introduction}

Dans le cadre des travaux de thèse (A. BAT 1999), nous avons tenté de discerner les influences, sur les caractéristiques mécaniques mesurés du sol, des différentes procédures depuis le mode de prélèvement jusqu'aưu conditions et procédures d'essais au laboratoire.

Le site de Cran a fait l'objet de nombreuses études dans le cadre de recherches programmées par les Laboratoires des Ponts et Chaussées. Les caractéristiques qui ont présidé au choix de ce site ont été une relative homogénéité d'ensemble puis une profondeur de 5 à $10 \mathrm{~m}$ de sédiments meubles, cohérents de consistance moyenne. De façon implicite, dans cette analyse nous avons supposé que le matériau prélevé et ausculté est homogène. Si cette présupposition est relativement vraie à l'échelle globale du site, une analyse fine du sol montre qu'il le parait moins dans le détail.

La plaine alluviale de la Vilaine en aval de Redon est une vallée sédimentaire de près de $2 \mathrm{~km}$ de large. On y rencontre un dépôt d'argile sur une épaisseur de 10 à $20 \mathrm{~m}$, reposant sur une couche de sable et de galets qui recouvre le substratum rocheux. A Cran,la rive droite est constituée par un dépôt d'argile molle marine de $17 \mathrm{~m}$ d'épaisseur reposant sur un substratum rocheux (schiste et phtanites). La couche d'alluvions compressibles est d'origine relativement récente. Elle est composée d'alluvions post-glaciaires, dont les périodes de formation s'échelonnent entre la période boréale ( 7500 ans) et la période subatlantique (2500 dernières années). L'argile de Cran contient environ 40 à $50 \%$ d'éléments inférieurs à $2 \mu \mathrm{m}$, la fraction fine étant surtout constituée de montmorillonite, d'illite et de kaolinite en proportion plus faible.

D'une manière générale, l'argile de Cran est grise et contient des fibres végétales (roseaux) presque toujours verticales. On distingue trois couches:

- les deux premiers mètres, surconsolidés par dessiccation, correspondent aux valeurs les plus fortes de cohésion non drainée, de l'ordre de $45 \mathrm{kPa}$;

- entre 2 et $4 \mathrm{~m}$ de profondeur, une couche de consistance relativement molle, de cohésion non drainée de l'ordre de $15 \mathrm{kPa}$, présente une forte limite de liquidité. Elle peut être identifiée, selon la classification $L P C$, à une argile très plastique et faiblement organique (fO-At);

- entre 4 et $17 \mathrm{~m}$ de profondeur, l'argile a une consistance moyenne; la teneur en eau est de l'ordre de $70 \%$ et la cohésion non drainée est voisine de $38 \mathrm{kPa}$. On peut identifier ce sol à un limon très plastique faiblement organique (fO-Lt). 
La nappe phréatique est généralement à $50 \mathrm{~cm}$ de profondeur. Le substratum rocheux est recouvert localement d'une couche d'environ 1,50 m d'épaisseur de graves pliocènes compactes.

Par ailleurs, on peut distinguer trois types de discontinuités ou facteurs d'hétérogénéité : la présence de débris végétaux, la présence de discontinuités sédimentaires et la présence de plans de fracturations et même de glissements.

\section{Hétérogénéité liée à la présence de végétaux}

Le milieu de dépôt correspond vraisemblablement à une plaine d'inondation avec immersions périodiques par des eaux chargées de particules argileuses. Ces inondations ou débordements se sont toujours produits, au moins, dans la zone d'étude, avec une énergie relativement faible puisque nous n'observons aucune particule grossière. Sur cette plaine s'est développée de façon peu intense une végétation essentiellement composée de roseaux.

On retrouve assez fréquemment dans les carottes des tiges de roseaux fines ou plus épaisses (de $3 \mathrm{~mm}$ à $1 \mathrm{~cm}$ de diamètre), et en section des restes de racines chargées de particules argileuses (figure 1). La présence de ces paléo - racines, de compacité plus élevée que le sol environnant, conduit probablement à une modification des paramètres mécaniques mesurés. Il est tout à fait probable qu'un certain nombre d'échantillons testés a contenu de tels débris sans que l'on s'en aperçoive.

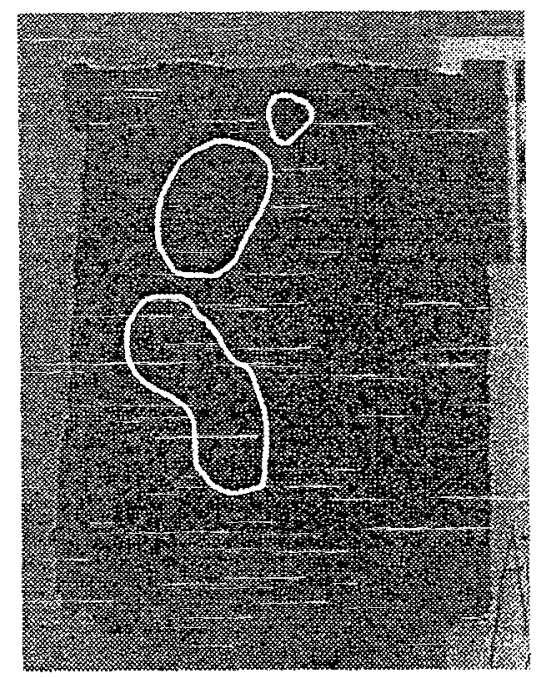

Figure 1. Photo d'une paléo-racine

\section{Les discontinuités sédimentaires}

Il s'agit de discontinuités acquises au moment du dépôt ou très peu de temps après celui-ci. Tout volume de matériaux sédimentaires se divise en volume plus petit (séries, bancs, lamines, etc.). Les surfaces séparant deux unités sont matérialisées par un contraste de faciès opposant les terres sous et sus-jacentes: les discontinuités peuvent être bien matérialisées (ravinements, encroûtements ) ou moins visibles (contraste de granulométrie). 
Certaines discontinuités correspondent à des périodes plus au moins prolongées d'émersion où la surface du sol a pu se durcir par dessiccation, et où la végétation a pu se développer.

D'autres discontinuités correspondent à des variations dans la nature des dépôts (variations de couleur ou zones enrichies de particules de mica, par exemple). Ces discontinuités peuvent être très rapprochées les unes des autres. On a pu observer sur un échantillon (sondage $J 7$ à $2,35 \mathrm{~m}$ de profondeur), un litage fin de couches de 1 à $2 \mathrm{~mm}$ d'épaisseur qu'il est plausible d'interpréter comme correspondant à des séquences de dépôts annuels (figure 2).

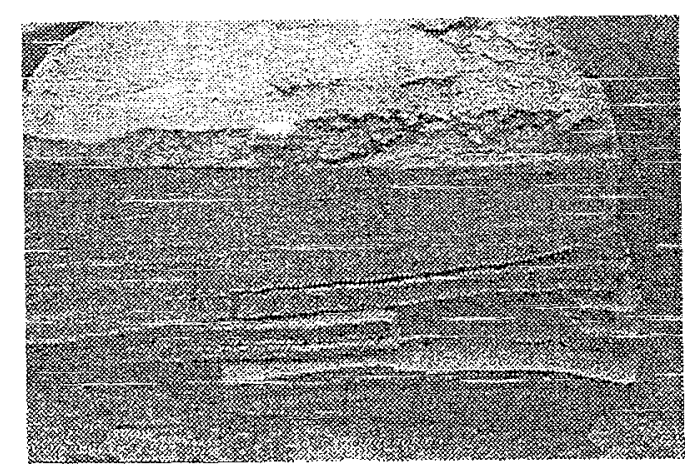

Figure 2. Photo d'un litage

Le caractère trop discontinu des observations nous a empêché de réaliser des corrélations de sédimentation, sondage à sondage, et de tenter des regroupements où chaque échantillon testé serait replacé dans une séquence sédimentaire, afin de comparer des échantillons de même séquence.

Il est cependant probable que des échantillons prélevés dans l'une où l'autre séquence, en particulier celles liées à des périodes différentes d'émersion peuvent avoir des comportements mécaniques différents. En outre, un échantillon possédant une discontinuité de ce type pourrait donner des résultats difficilement interprétables.

\section{Les discontinuités de fracture et de glissements}

\section{a - Description}

Dans de nombreuses carottes, nous avons pu observer des surfaces de glissements (figure 3) faisant avec l'horizontale des angles de l'ordre de 40 à 45 degrés, avec des déplacements soit en "failles inverses" soit en "failles normales". Ces surfaces sont le plus souvent rectilignes et parfois courbées avec une diminution de l'angle vers le bas.

Ces plans de glissements apparaissent assez nombreux ; ainsi sur les 4 prélèvements du sondage G6, six plans de ce type ont été repérés, parfois proches les uns des autres $(2,4 \mathrm{~m} ; 4,8 \mathrm{~m} ; 7,45 \mathrm{~m} ; 7,77 \mathrm{~m} ; 8,05 \mathrm{~m}$ et $8,45 \mathrm{~m})$.

Au total, sur l'ensemble des échantillons testés lors du programme de recherche dans le cadre des travaux de BAT (1999), (43 échantillons soit une longueur totale de $30 \mathrm{~m}$ ) 21 fractures de ce type ont été décelées (dans ce décompte, les fractures décelées lors de la taille ne sont comptabilisées). 11 est à noter que ces plans de 
glissements n'ont été décelés que sur les sondages $\mathrm{G}, \mathrm{H}$ et I (figure 4). Soit sur 24 échantillons, nous avons remarqué 21 fractures. Cela indique une fréquence importante de ces plans près de la berge et pourrait ainsi privilégier la thèse de la décompression latérale, plus ou moins liée à des plans de glissements de berge, comme origine de ces fracturations.

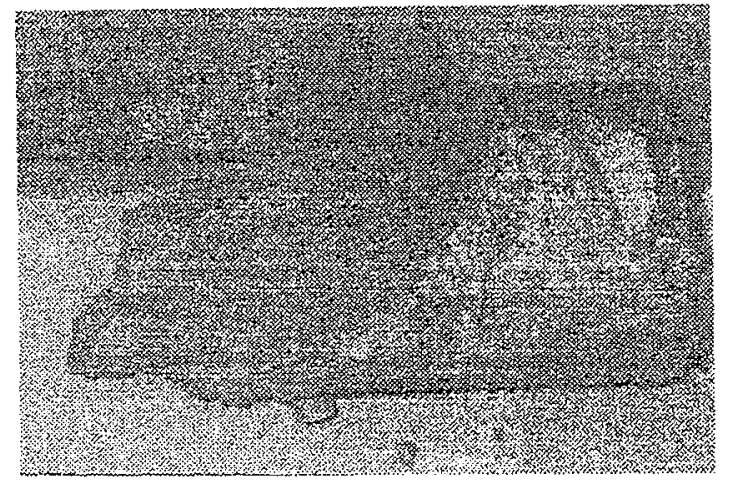

Figure 3. Exemple de plan de glissement observé

En outre nous avons pu observer sur le sondage $\mathrm{H} 7$ entre $5 \mathrm{~m}$ et $5,2 \mathrm{~m}$ une zone de $3 \mathrm{~cm}$ environ inclinée d'un angle de $40^{\circ}$ qui semble plus compacte que le sol environnant. Nous avons effectué des mesures de la teneur en eau sur cette zone et sur les matériaux immédiatement au-dessus et immédiatement au-dessous de cette zone plus compacte. Les résultats de ces mesures montrent effectivement une différence notable d'état entre d'une part la zone compacte inclinée de teneur en eau $68 \%$, et d'autre part, les matériaux environnants de teneur en eau de $91 \%$, soit un écart de teneur en eau de l'ordre de 23 points. On peut se demander si cette zone ne comespond pas à l'amorce d'un plan de glissement du type de ceux que l'on a pu observer sur d'autres carottes. On a pu observer également qu'assez souvent ses discontinuités s'arrêtaient sur des discontinuités sédimentaires.

\section{b - Interprétations génétiques}

Le contexte géologique de la formation étudiée, (des sédiments alluviaux très récents, dans un secteur tectoniquement stable) interdit une interprétation de ces fractures comme étant consécutives à des mouvements tectoniques.

Fracturation par décompression latérale

La décompression latérale de la vallée de la vilaine pourrait être considérée comme une interprétation possible de l'origine de ces fractures. L'argument en faveur de cette hypothèse est que les sondages les plus affectés sont ceux proches de la vallée. Néanmoins, cette vallée reste topographiquement peu marquée et la hauteur hors de l'eau n'excède pas aujourd'hui $5 \mathrm{~m}$ (figure 5). Si ce phénomène a pu se produire, il n'a probablement pas pu seul être à l'origine de cette fracturation. En outre cette interprétation génétique ne peut expliquer l'observation en failles inverses visibles clairement dans certains échantillons. 


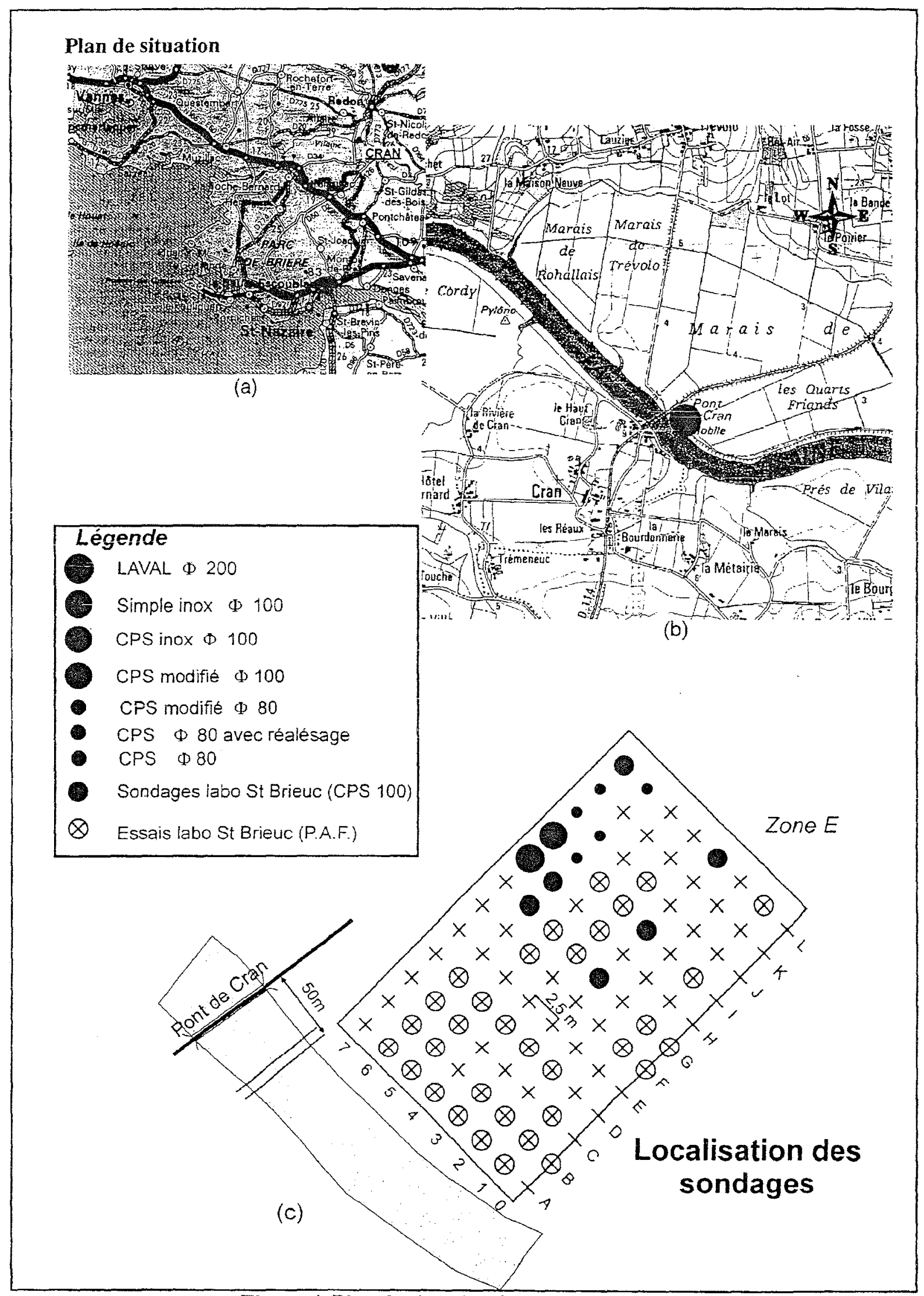

Figure 4. Plan de situation Site de Cran 


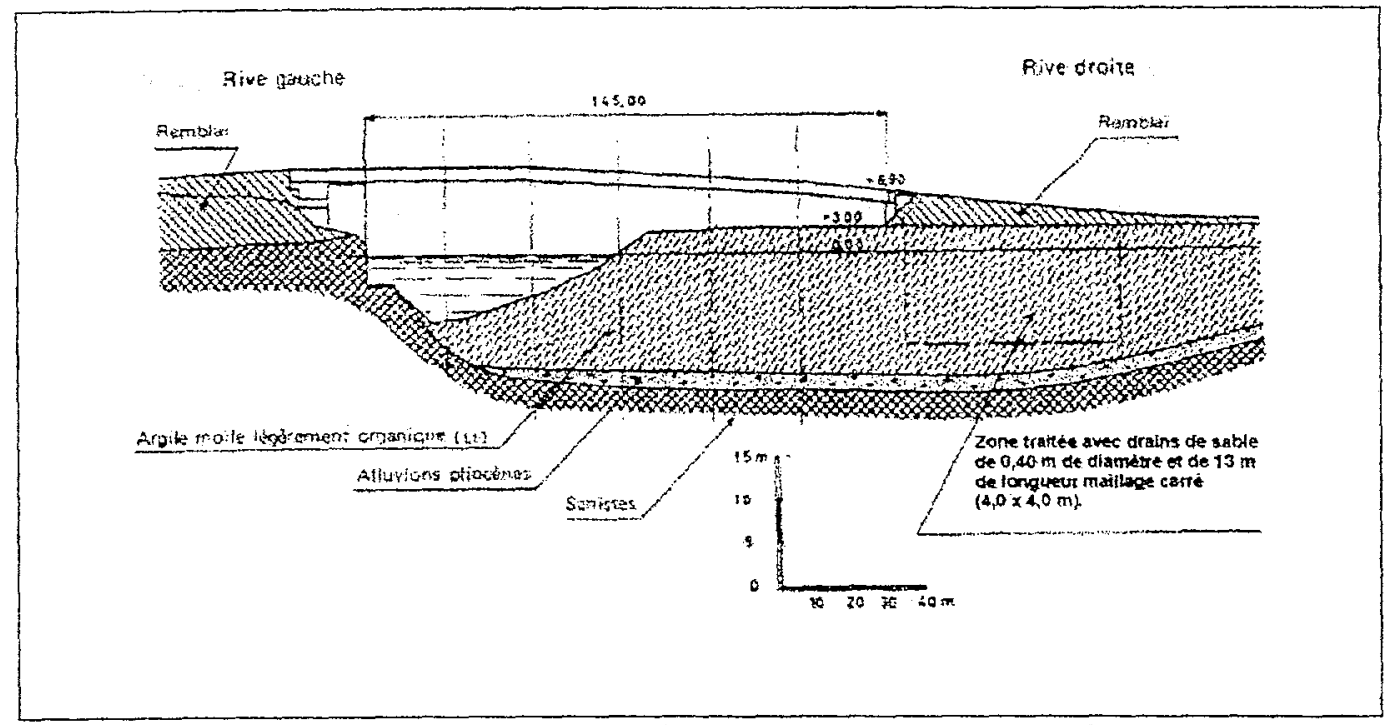

Figure 5. Coupe géologique sommaire du site de Cran (profil en long du pont de Cran)

d'après Paute (1973)

Fracturation par compaction

La compaction verticale subite par ces terrains depuis leur état initial (vase) jusqu'à leur état actuel est loin d'être négligeable. En effet, si on considère que le matériau initial avait une teneur en eau de $200 \%$ (ordre de grandeur des teneurs en eau dans les sédiments portuaires actuels) et une masse volumique des grains solides $\rho_{\mathrm{s}}=2,52 \mathrm{Mg} / \mathrm{m}^{3}$ on obtient un indice des vides initial $\mathrm{e}_{\mathrm{o}}=(2 * 2,52)=5,04$ $\mathrm{d}^{\prime}$ où une masse volumique sèche initiale $\rho_{\mathrm{do}}=0,417 \mathrm{Mg} / \mathrm{m}^{3}$. Ce matériau possède actuellement des teneurs en eau comprise entre $70 \%$ et $110 \%$. En considérant une teneur moyenne de $90 \%$ on obtient un indice des vides $e_{\mathrm{f}}=2,27$ et une masse volumique sèche actuelle $\rho_{\mathrm{df}}=0,771 \mathrm{Mg} / \mathrm{m}^{3}$. Ainsi une couche de sol de hauteur initiale de $1 \mathrm{~m}$ a actuellement une hauteur $\mathrm{H}_{\mathrm{f}}=0,54 \mathrm{~m}$ avec un rapport $\mathrm{H}_{\mathrm{f}} / \mathrm{H}_{\mathrm{o}}=0,54$.

Par ailleurs nous avons pu noter, dans le sondage I6, un roseau entre $4,90 \mathrm{~m}$ et $5,05 \mathrm{~m}$ présentant de nombreux plis. Nous avons interprété cette figure comme étant la conséquence de la compaction qu'a subit le sol. La mesure en ligne droite du point haut au point bas du roseau donne une longueur de $15,5 \mathrm{~cm}$; sa longueur curviligne est de $26,5 \mathrm{~cm}$. Le rapport entre ces deux longueurs est de 0,58 . Ce roseau se situe dans des terrains où la teneur en eau est de l'ordre de $90 \%$, on peut noter la bonne cohérence entre l'évaluation du taux de compaction du calcul approché ci-dessus et l'observation faite sur le roseau. On peut penser que, lors de la consolidation sous le poids propre des terrains, la contrainte verticale induit des discontinuités à $45^{\circ}$ probablement peu continues car s'arrêtant sur des discontinuités sédimentaires horizontales. L'eau va s'évacuer plus rapidement dans ces zones de discontinuité que dans les zones homogènes. Ceci expliquerait l'observation des variations de la teneur en eau mesurées sur l'échantillon situé à $5,1 \mathrm{~m}$ du sondage $\mathrm{H} 7$. Le phénomène va donc avoir tendance à s'accentuer pour finalement créer une diaclase (fracture sans déplacement) qui aura un angle d'environ $45^{\circ}$. Lors de la consolidation, le comportement du massif argileux n'est 
pas homogène horizontalement du fait que le substratum n'est pas horizontal et / ou n'est pas régulier. Il peut se produire des tassements différentiels. Ces tassements vont être à l'origine de déplacements latéraux le long de ces fractures qui pourront jouer en failles normales ou en failles inverses en fonction de l'orientation de la fracture et de la disposition des tassements différentiels par rapport à ces fractures (figure 6). Ces fractures étant elles-mêmes la résultante de ce processus de compaction, il est tout à fait logique qu'elles puissent présenter des formes courbes.

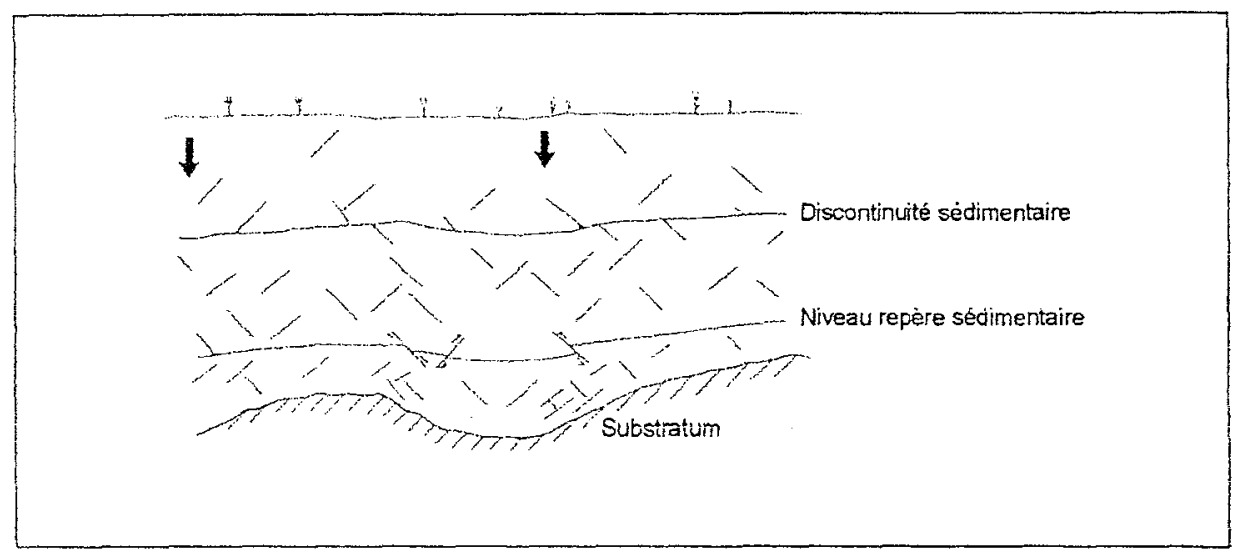

Figure 6. Schéma explicatif possible des fracturations par compaction

\section{Conclusion}

L'analyse géologique faite sur le massif argileux de Cran a mis en évidence une forte variabilité. Cette variabilité provient des techniques de prélèvement, pour une partie, mais aussi de la variabilité spatiale naturelle du massif argileux de Cran. La genèse du site permet de l'expliquer. En effet, les argiles molles de Cran ont déjà une histoire, source de discontinuité et d'hétérogénéité. Ces discontinuités sont liées, d'une part, au mode de dépôt, et d'autre part probablement liées au processus de sédimentation et de consolidation qui n'est pas négligeable, puisque l'épaisseur des vases originelles a été finalement réduite d'au moins de 2 . Les nombreux plans de glissement à $40-45$ degrés observés dans les prélèvements, sont les conséquences de ce processus et des glissements de berges. Ces glissements se produisent suite à l'évolution des estuaires dans le temps. Il est clair que ces discontinuités d'origine sédimentaire ou dynamique sont à l'origine de l'hétérogénéité du site. Ceci peut rendre moins surprenant de constater que des échantillons prélevés à la même profondeur ont des propriétés physiques différentes et des comportements mécaniques différents.

\section{Bibliographie}

BAT A. (1999) "Incidence des procédures de prélèvement et des procédures d'essais sur les paramètres géotechniques mesurés". Thèse de doctorat en génie civil, Université de Caen, 185 p., Annexes 235 p.

PAUTE J.L. (1973) "Etude des tassements. Remblai de Cran".

Bulletin des laboratoires des Ponts et Chaussées Spécial T, Mai, pp. 105-118. 